\title{
¿La innovación social como método de investigación participativo y sociopráctico?
}

\section{Social innovation as a participatory and socio- practical research method?}

\author{
José Hernández-Ascanio \\ Universidad de Córdoba, España \\ jhascanio@uco.es
}

\begin{abstract}
Resumen: La innovación social se ha constituido en uno de los ámbitos de estudio más activos no sólo en el campo de investigación sobre la innovación, sino de las ciencias sociales en general. Desafortunadamente, ese interés no ha conducido al desarrollo de una teoría de la innovación social.

En la actualidad es posible identificar un intenso debate teórico en torno a tres áreas de interés fundamentales: la formulación de definiciones y conceptos sobre qué es la innovación social, la identificación de las etapas a partir de las cuales discurren los procesos de innovación social y, por último, el intento de elaborar modelos integradores sobre dichos fenómenos.

Se pueden constatar diferentes esfuerzos de articulación de estos elementos focales. Uno nuevo que se abre es la reflexión sobre el carácter praxeológico de la innovación social, más allá de un conjunto de metaasunciones, elementos explicativos $\mathrm{u}$ objetivos de investigación. En la innovación social se reconocen significativos paralelismos con los modelos investigación participativa y de sociopraxis, de tal forma que se hace necesario elaborar la pregunta acerca de si es posible considerar la innovación social como un método propio de este tipo de metodología de indagación colectiva.

El presente trabajo propone una discusión en este sentido, utilizando para ello una estrategia hermenéutica aplicada a una revisión sistemática e integrativa de la bibliografía especializada. En el desarrollo disciplinar en torno al fenómeno de la innovación social es posible identificar fundamentos epistemológicos que permiten caracterizarlo como un método propio de investigación participativa y de sociopraxis social específica para la generación de productos culturales de alto impacto social. Sin embargo, la dispersión y la debilidad conceptual y metodológica en torno a la innovación social se presentan como principal obstáculo para la consolidación de la misma como método.
\end{abstract}

Palabras clave: Innovación social, metodología, métodos participativos. 


\begin{abstract}
Social innovation has become one of the most active fields of study not only in the field of research on innovation but also in the social sciences in general. Unfortunately, that interest has not led to the development of a theory of social innovation.

Currently, it is possible to identify an intense theoretical debate around three fundamental areas of interest: the formulation of definitions and concepts about what social innovation is, the identification of the stages from which social innovation processes run and, finally, the attempt to elaborate integrative models on these phenomena.

Different articulation efforts of these focal elements can be verified. A new one that is opening is the reflection on the praxeological character of social innovation, beyond a set of meta-assumptions, explanatory elements or research objectives. In social innovation, significant parallels are recognized with the participatory research and sociopraxis models, in such a way that it is necessary to elaborate the question about whether it is possible to consider social innovation as a method of this type of collective inquiry methodology .

The present work proposes a discussion in this sense, using a hermeneutical strategy applied to a systematic and integrative review of the specialized bibliography. In the disciplinary development around the phenomenon of social innovation, it is possible to identify epistemological foundations that allow it to be characterized as its own method of participatory research and specific social sociopraxis for the generation of cultural products with high social impact. However, the dispersion and conceptual and methodological weakness around social innovation are presented as the main obstacle to consolidating it as a method.
\end{abstract}

Keywords: Social innovation, methodology, participatory methods. 


\section{INTRODUCCIÓN}

La incapacidad a la hora de definir claramente la innovación social ha demostrado ser un obstáculo central a la hora de establecer una agenda de investigación progresiva en este campo. En trabajos previos (Hernández-Ascanio et al., 2016) ha sido posible demostrar que, a pesar de que el interés por investigar en el ámbito de la innovación social se fortalece, dicha investigación se realiza de una forma altamente fragmentada y separada de las prácticas sociales (Marques et al., 2012; Nicholls y Murdock, 2012; Kirwan, Ilbery, Maye, \& Carey, 2013; Nicholls, Simon, \& Gabriel, 2016; Cajaiba-Santana, 2014).

Desafortunadamente, esa profusión por el interés en torno a la innovación social no ha conducido, hasta el momento, al desarrollo de una teoría de la innovación social. (Nicholls et al., 2016; Tepsie, 2014; Pot y Vaas, 2008; Adams y Hess, 2010; Howaldt y Schwarz, 2010; Hochgerner, 2011; Marques et al., 2012; Cajaiba-Santana, 2014; Murray et al., 2010; Bassi, 2011). A pesar de todo ello, es preciso llevar a cabo un esfuerzo de articulación de estos elementos focales para tratar de extraer los elementos más significativos que permitan sentar las bases de una fundamentación teórica sobre la innovación social.

En este esfuerzo, es posible proponer un enfoque novedoso de articulación epistemológica, metodológica y conceptual sobre el fenómeno de la innovación social. Esta nueva vía es la de discutir la innovación social como un método de investigación-indagación participativa/colaborativa, así como de sociopraxis especializada en la génesis de productos culturales de alto impacto social, y no sólo como un conjunto de metaasunciones, elementos explicativos y objetos de investigación (Portes, 2013). En esta pretensión, este trabajo va a seguir muy de cerca la propuesta analítica de Caballero Ferrándiz et al. (2019). Este trabajo asume junto con estos autores que en las metodologías y métodos participativos la cuestión de primer orden para tener en cuenta no es tanto el elemento técnico, sino la intencionalidad y la posición ética con respecto a los procesos de cambio social de los actores que intervienen como facilitadores e investigadores (Callejo y Viedma, 2006; Cimas, 2015).

En la medida en que la investigación con enfoques participativos se construye con una lógica de paradigma, hay afectación sobre el uso instrumental de las técnicas, sea cual sea su naturaleza en la medida en que se ven afectadas en su diseño por la construcción situada de los actores sociales, ya que estaríamos situándonos con una investigación de carácter intencional, es decir «una investigación para la acción concreta» (Ca1lejo y Viedma, 2015). 
El presente trabajo aspira a avanzar en esta línea de discusión, ya que se ha identificado como un gap a cubrir dentro de la agenda de investigación sobre esta temática. Para ello, se discutirá, a partir de un enfoque hermenéutico, si los diferentes enfoques existentes sobre innovación social reúnen los fundamentos epistemológicos necesarios para poder ser considerados como un modelo de sociopraxis social y de investigación/ indagación participativa-colaborativa. Este trabajo abordará como fundamentos epistemológicos de discusión cuatro elementos: 1) la fundamentación científica de la innovación social; 2) el carácter colaborativo de la innovación social; 3) el componente crítico-político presente en la innovación social; y 4) el carácter de proceso presente en los procesos de innovación social.

En lo que refiere al análisis de la dimensión procesual de la innovación social, es posible identificar en la bibliografía un amplio vacío, por lo que se ha optado por llevar a cabo la discusión a partir del Modelo Sistemático de Capacidades de Innovación Social, que es una propuesta original del autor como resultado de una sistematización y consolidación integrativa de otros modelos presentes en la literatura científica.

La estructura del presente artículo responde a estos dos momentos que se han señalado, correspondiéndose los epígrafes con un primero, que sirve como preámbulo, y en el que se hace una presentación general de los principales componentes teóricos y metodológicos asociados a la innovación social. El segundo epígrafe se dedica a discutir los diferentes enfoques de innovación social como método de sociopraxis y de investigación/indagación colectiva y participativa a partir de sus principales fundamentos epistemológicos. El tercer epígrafe se dedicará a discutir el fundamento epistemológico específico del proceso, por ser una de las principales deficiencias identificadas en la bibliografía. Para llevar a cabo esta discusión se utilizará como referencia la propuesta analítica de Caballero Ferrándiz et al. (2019), que se aplicará al Modelo Sistemático de Capacidades de Innovación Social. El trabajo se cierra con un epígrafe dedicado a establecer las principales conclusiones obtenidas.

\section{Hipótesis y objetivos}

Este trabajo parte de la hipótesis afirmativa de que la innovación social reúne las condiciones epistemológicas y metodológicas potenciales para ser considerado como un método de socio-práxis social y de investigación e indagación participativa-colaborativa especializada en la génesis de productos culturales de alto impacto social, aunque aún no se han dado los desarrollos disciplinares suficientes para que se consolide como tal.

El objetivo principal de este trabajo es discutir si el fenómeno de la innovación social reúne las condiciones necesarias para poder ser considerado como un método de sociopraxis social y de investigación/indagación participativa y colaborativa. 


\section{Metodología}

Para proporcionar una descripción general de la base de conocimientos, este trabajo ha realizado una revisión sistemática de la literatura en combinación con una revisión narrativa como método validado de generación de conocimiento (Denyer y Neely, 2004; Pittaway et al., 2004; Tranfield et al., 2003).

Para definir los parámetros de la revisión, así como la selección de algunos elementos conceptuales de primer orden, tales como las «capacidades de innovación social», se estableció un panel DELPHI compuesto por catorce expertos en metodología y teoría, así como destacados profesionales que operan en los campos de estudio, en este caso, la innovación social. Se diseñó un protocolo de revisión por la dirección para respaldar el proceso que era suficientemente flexible para permitir la creatividad, pero suficientemente estructurado para evitar cualquier sesgo del investigador que afectase al resultado (Tranfield et al., 2003).

Se realizó una búsqueda inicial de documentos en diferentes bases de datos de referencia (ISI Web of Knowledge, SCOPUS), para el periodo 2000 a 2019.

El equipo de revisión identificó temas sobre la materia en función de sus antecedentes y experiencia, desarrollando estos temas en un marco de análisis que consideró los siguientes: «innovación social»y otras combinaciones como: «se define la innovación social», «definición de innovación sociales», «innovación social se refiere a», «innovación social es conceptualizada», «dimensiones de la innovación social» y «la investigación sobre la innovación social».

Se consideraron el inglés y el castellano como idiomas preferenciales de publicación. Un primer resultado de esta búsqueda arrojó un total de 1.986 registros. Sobre este catálogo inicial se decidió aplicar un filtro más restrictivo consistente en que en el cuerpo del artículo apareciese la definición explícita de la innovación social. Utilizando este criterio se redujo la relación a un total de 62 artículos, que dieron lugar a 48 definiciones diferentes.

Para complementar la información anterior se procedió a realizar una nueva búsqueda flotante en Google Scholar y la literatura gris que incluía libros, artículos, documentos de trabajo e informes citados en orden decreciente ING y editoriales y tesis excluidos.

Para reducir el número de citas, los títulos de los artículos se analizaron según los criterios de exclusión, y luego se analizaron los resúmenes según los criterios de inclusión. Para llevar a cabo esta selección se han utilizado criterios de citación (aquellas definiciones que se incorporan en obras posteriores), relevancia (aquellas que dan lugar a marcos teóricos más o menos elaborados) y genuinidad (es decir, que aporta elemen- 
tos de gran impacto, ya sea por el hecho de que se han consolidado dentro de escuelas de pensamiento o bien por pertenecer a instituciones de referencia en el ámbito de la innovación social).

A partir de sistematizaciones llevadas a cabo tanto en el ámbito de las definiciones y de las modelizaciones se ha identificado una serie de dimensiones comunes con la intención de utilizarlas como recursos en una taxonomía de dicho fenómeno (Wang y Wang, 2003). Las dimensiones identificadas son:

- Gestor de la innovación social, o actor principal encargado de liderar el proceso de innovación social.

- Objeto principal de la innovación social.

- Capacidad de transformación social, que va a tener que ver con la capacidad de producir cambios en más de un ámbito social interconectando diferentes esferas sociales. Cuanto más profundo y difundido sea el cambio social, mayor será la capacidad de transformación social.

- El ciclo de proceso, entendido esto como la reflexión y sistematización acerca de los diferentes elementos y acciones que intervienen o deben estar presentes en un proceso de innovación social.

- Implicación disciplinar o dimensión predominante, que tiene que ver con la importancia dada a alguno de los elementos económicos, políticos o sociales dentro de la propia definición.

A partir de estos elementos se procederá en el siguiente epígrafe a discutir si los diferentes enfoques que existen en el ámbito de la innovación social reúnen los fundamentos necesarios para ser considerados un método de investigación/indagación participativa y colaborativa y de sociopraxis social.

\section{¿ES LA INNOVACIÓN SOCIAL UN MÉTODO DE SOCIOPRAXIS?}

Tanto los denominados métodos de investigación-acción como los de socio-práxis se pueden encuadrar dentro de los enfoques cualitativistas. La principal característica de este enfoque es la relación cercana y simétrica entre los diferentes actores que participan del proceso (investigadores e investigados), así como el uso de aproximaciones de carácter hermenéutico (Chaves, 2007).

En este tipo de procesos, el conocimiento es resultado de una estrategia colectiva y colaborativa en la que se negocian los sentidos y significados subjetivos sobre la reali- 
dad, de tal forma que la realidad social es el resultado de esta negociación, inserta en el contexto de cotidianidad de los participantes (Chaves, 2007).

Con respecto a la definición concreta de la investigación-acción propiamente dicha o de la sociopraxis no existe una unanimidad (Colás-Bravo, 1998), aunque sí es posible identificar una serie de elementos comunes.

En primer lugar, estaría la idea de que se trata de métodos basados en prácticas sistemáticas cuyo objetivo es la construcción colaborativa de conocimiento y de forma de acceso al mismo, donde no hay distinción entre sujeto y objeto del acto de conocer (Chaves, 2007).

Una segunda característica para tener en cuenta es que, en este tipo de métodos, la construcción de conocimiento no representa nunca una labor unívoca ni unidireccional.

Un tercer elemento es que el componente crítico representa a su vez un necesario componente trasversal que se expresa a partir de cuatro procesos básicos: 1) está orientado hacia la transformación y mejora de las prácticas sociales disponibles; 2) es producto de un desarrollo participativo y colaborativo; 3) se desarrolla mediante un proceso sistemático de producción; y 4) supone un proceso de aprendizaje continuo por parte de los propios actores implicados (Colás-Bravo, 1998).

Un cuarto elemento a tener en cuenta es que este tipo de métodos precisan de un proceso sistemático de acción que está claramente definido (Colás-Bravo, 1998).

\section{Fundamentos epistemológicos de la innovación social como método de investigación-indagación participativa/colaborativa y de sociopraxis social}

En el presente apartado se abordan lecturas posibles de la innovación social como método de investigación participativa-colaborativa y de sociopraxis. De esta forma, se realizan valoraciones sobre diferentes aspectos claves de dicha metodología a partir de las características expresadas anteriormente, a fin de sustentarla epistemológicamente. Dicho de otra forma, se pretende sustentar un enfoque metodológico desde la subdisciplina epistemológica.

Este epígrafe se encuentra desglosado en varios temas específicos. El primero tendría que ver con la fundamentación científica de la innovación social a partir de la identificación de su objeto de interés. El segundo tema a tener en cuenta sería el carácter colaborativo de dicho método desde la dimensión «gestor de la innovación social o agente» que ya se ha indicado anteriormente. El tercer tema sería el componente crítico-político presente en la innovación social a partir de la dimensión «Capacidad de transformación social», para, por último, abordar el carácter de proceso presente en los procesos de innovación social desde la dimensión «Ciclo de proceso». 
a) La dimensión «Objeto principal de la innovación social».

La unión de dos conceptos tan flexibles y polisémicos como innovación y social da como resultado que las definiciones de innovación social hayan adoptado matices tan diferentes. A esto hay que añadir el hecho de que existe multitud de términos dentro de la propia definición (mejorar la calidad de vida, cambio social, etc.) que también precisan de una definición más concreta (Anderson et al., 2014; Cunha y Benneworth, 2013; Edwards-Schachter et al., 2012; Bignetti, 2011; Cajaiba-Santana, 2014).

La revisión sistemática llevada a cabo permite decir que, desde el punto de vista del análisis paradigmático, se identifican dos grades escuelas: la que identifica a la innovación social desde una orientación de carácter mayoritariamente tecnocrático y la que da mayor relevancia a la dimensión democrática.

La primera orientación tiene su fundamentación claramente en planteamientos de carácter neoliberal, y aunque promueve un discurso orientado al empoderamiento comunitario, en la práctica nos encontramos con que sus acciones tienden a valorizar el papel de los expertos y a movilizar tecnologías tanto de gobierno como de política pública, todo ello con el objetivo de reducir en la medida de lo posible cualquier tipo de disidencia de carácter político. Tras un discurso aparentemente alineado con la participación, se identifican prácticas que están orientadas realmente a fortalecer una distribución jerárquica de carácter vertical del poder en el ámbito de la sociedad.

Por su parte, la orientación de carácter democrática, cuyas posiciones tienen como objetivo proponer modelos alternativos al neoliberalismo, no se reduce a la promoción de la participación comunitaria, sino que también reivindica todo un proceso de puesta en valor del conocimiento producido comunitariamente, otorgándole el mismo valor que al conocimiento experto (Moulaert et al., 2013). Además, esta orientación democrática parte de la presunción de que la innovación social puede servir como estrategia óptima con la que recuperar en los espacios sociales el debate político neutralizado por los planteamientos neoliberales, promoviendo de esta manera una distribución horizontal y colaborativa del poder en la sociedad.

b) La dimensión «Gestor de proceso» o «Agente en los procesos de innovación social».

Centrándose en cada una de las dimensiones que se han planteado en el anterior epígrafe, se ve que en lo relativo a la dimensión «agente» se detectan claras correspondencias entre definiciones que identifican a los emprendedores sociales como agentes más significativos y las definiciones que plantean que el contenido de la innovación 
social es la cobertura de necesidades sociales. Estas definiciones también se caracterizan por ser las que prestan mayor interés a la dimensión económica en detrimento de la capacidad de transformación social. De igual manera, es posible identificar una correlación entre aquellas formulaciones teóricas que sitúan el foco de atención en promotores de carácter institucional, las que consideran los contenidos de las innovaciones sociales en términos de cobertura de demandas sociales que además tienen una capacidad media de transformación social y las caracterizadas por orientaciones de carácter gerencial o politológico.

En las definiciones que parten de un papel más protagónico de la sociedad civil organizada, los contenidos de la innovación social son más flexibles y diversos (desde la cobertura de necesidades sociales hasta procesos de profunda transformación social) y la capacidad de transformación y la importancia dada a los procesos de participación-inclusión de agentes sociales son de carácter elevado.

Se identifica una evolución de la conceptualización sobre la innovación social, en cuanto a la dimensión agente, entre polos en los que se sitúan los emprendedores sociales en exclusividad a una presencia coral de actores conformada por sociedad civil organizada, Administración, etc., y que se relacionan en el marco de lo que se ha venido a denominar como «ecosistemas de innovación social».

Cuanta mayor diversidad se encuentra entre los agentes responsables de gestionar los procesos de innovación social, más plurales y heterogéneos serán los contenidos de éstos, asumiendo tanto la cobertura de necesidades sociales como la respuesta a demandas socialmente definidas o la implementación de procesos de cambio social en mayor o menor profundidad.

c) La dimensión «Capacidad de Transformación Social».

La línea principal de los estudios relacionados con la innovación social es la que aborda los efectos de este tipo de innovación en la sociedad (Frank Moulaert \& Nussbaumer, 2005; Hochgerner, 2011; Jessop et al., 2013; Subirats y García Bernardos, 2015). A pesar de esta presunción, la revisión de la literatura científica desarrollada en este campo da cuenta de los escasos estudios empíricos que han tratado en profundidad este aspecto.

En general, a pesar de esta situación, se cuenta con algunas evidencias provenientes de estudios de casos que apuntan a que la innovación social es un determinante de la transformación de la sociedad, toda vez que genera valor social (Pot \& Vaas, 2008; Edwards-Schachter et al., 2012). 
En esta línea, los trabajos de Frank Moulaert \& Nussbaumer (2005) demuestran que la innovación social potencia el desarrollo territorial, al modificar favorablemente las relaciones entre los individuos, dentro y entre los grupos nuevos o ya establecidos, al tiempo que fomenta la creación de canales de comunicación entre las organizaciones y su entorno (Marcy \& Mumford, 2007).

Del mismo modo, se afirma que la innovación social favorece la asimilación de nuevas creencias y modelos mentales para el desarrollo, tanto en la organización como en su entorno (Pot \& Vaas, 2008; Edwards-Schachter et al., 2012). Algunos trabajos han confirmado la alta influencia de la innovación social en la creación de nuevas estructuras sociales y la adopción y cambio de conductas en el estilo de vida de las personas (Hubert, 2010).

Recientemente, los trabajos de grupo de Tepsie (2014) señalan que la innovación social potencia la creación de nuevas formas de relación y de ejercer el gobierno, visibilizando y promoviendo a nuevos actores con potencial para desarrollar ideas nuevas y más eficaces con impacto social. Para este grupo de estudio, la innovación social genera nuevas formas de colaborar y de participar, diluyendo los papeles tradicionales de unos y otros, y borrando las líneas funcionales de los productores, compradores y usuarios que hasta ahora venían estando muy delimitadas (Tepsie, 2014; Neumeier, 2012).

Otros trabajos señalan que la innovación social tiene la habilidad para fortalecer nuevas capacidades en el conjunto de actores y de optimizar los recursos actuales y/o latentes, sobre todo, en la intersección de sectores, llegando a facilitar la creación de un ecosistema con potencial para generar organizaciones híbridas (Frank Moulaert \& Nussbaumer, 2005; Hochgerner, 2011; Jessop et al., 2013; Subirats y García Bernardos, 2015), y es justo la combinación de estos elementos lo que confiere a la innovación social su carácter eminentemente colaborativo, característica que aumenta de manera determinante su potencial para dar respuesta, de manera realista, a las necesidades sociales movilizando el componente más innovador así como los mejores recursos y capacidades de cada uno de los actores implicados.

d) La dimensión «Ciclo de proceso».

Una cuestión importante es la ausencia de desarrollos teóricos sobre las diferentes etapas de la innovación social y las cuestiones de carácter procesual a la que están vinculadas. Mayoritariamente, las definiciones obvian este aspecto y los diferentes intentos de teorización se limitan a repetir o trasladar «etapas» o «fases» indicadas por autores de referencia sin profundizar en las mismas, en sus condiciones o en sus vinculaciones. Salvo raras excepciones, las definiciones no conceden un especial interés al proceso o 
procesos por los cuales se produce la innovación social, asumiendo de manera implícita que la innovación social es un fenómeno que emerge casi por generación espontánea, lo cual dificulta un intento de sistematización de dichos fenómenos.

No obstante, frente a que esta falta de interés por un análisis de las fases o etapas de la innovación social es algo bastante generalizable en la mayoría de las formulaciones de esta, sí es posible encontrar reflexiones sobre esta cuestión en particular y formulaciones más influidas claramente por los principios de la innovación clásica y cuyos contenidos están claramente delimitados (la cobertura de necesidades sociales, por ejemplo).

Como resulta una cuestión de primer orden poder analizar los procesos de innovación social en paralelo a la sistematización de otros métodos de investigación participativa-colaborativa y de sociopraxis, por ser uno de los principales gaps identificados en la bibliografía, se procederá a dedicar un epígrafe completo a dicha cuestión a continuación.

\section{LOS MÉTODOS DE SOCIOPRAXIS COMO PROCESOS EN 8 SALTOS}

Siguiendo el marco analítico propuesto por Caballero Ferrándiz et al. (2019), es posible identificar una serie de elementos secuenciales o «saltos» que permiten diferenciar procesos enraizados en las dinámicas de sociopraxis de otras dinámicas de producción social de carácter más aleatorio y espontáneo.

Algunas precisiones que hay que tener en cuenta según este autor con respecto a esta estrategia analítica son básicamente tres. En primer lugar, que los momentos secuenciales o saltos no deben ser confundidos con fases o etapas dentro un proceso, ya que no ocurre en la dinámica de sociopraxis. En segundo lugar, que los elementos secuenciales no son simplemente referencias de un itinerario, sino que suponen transformaciones sustantivas del sistema social de referencia en el que tiene origen la innovación social. Así mismo, hay que tener en cuenta que supone un proceso en el que se van alternando y complementando lógicas, metodologías y acciones que tienen un finalidad sumativa e integrativa. Junto con esto, y en último lugar, hay que entender que supone la concurrencia de enfoques, acciones y productos en un mismo soporte.

Se van a enunciar cuáles son los principales rasgos determinantes de cada salto, para posteriormente ponerlos en relación con los elementos expuestos del Modelo Sistemático de Capacidades de Innovación Social a fin de discutir la posibilidad de ser valorado o no como método de sociopraxis. 
- Salto 1: La autorreflexión del equipo y del grupo motor.

La autorreflexión del equipo motor es un prerrequisito de cualquier dinámica de facilitación de procesos en el que los implicados toman consciencia y hacen explícitas las construcciones mentales de las que parten con respecto al proceso que quieren iniciar. Aunque es una cuestión de primer orden, en la medida en que muchos de los nudos y dificultades de los procesos de cocreación de conocimiento se encuentran en la dificultad para armonizar e integrar las diferentes construcciones mentales, es un reto que por cuestiones de agenda $\mathrm{u}$ otros motivos suele obviarse.

- Salto 2: La negociación inicial y el plan de trabajo.

Es el contexto en que no sólo se definen de manera colaborativa los objetivos, los instrumentos, la hoja de ruta del proceso, sino que también se constituye el grupo motor como un «grupo inteligente»(Cembranos \& Medina, 2003; Vercauteren et al., 2010), en el que se establecen vínculos afectivos y operacionales con niveles suficientes de eficacia.

- Salto 3: Mapas estratégicos y conjuntos de acción.

El tercer salto hace referencia al contexto en que el grupo motor elabora un «mapeo de relaciones entre actores», un «mapa estratégico» o «sociograma con conjuntos de acción»(CIMAS, 2015). Este momento supone ir más allá de listar los diferentes recursos tangibles o intangibles presentes en el contexto, ya sean favorables o no a la acción. Realmente lo que se precisa es establecer un modelo tridimensional de agentes, recursos y dinámicas de interacción entre los mismos y con elementos externos al sistema que puedan actuar como determinantes o condicionantes de la posibilidad de acción.

— Salto 4: Escuchar más allá de los dilemas dominantes.

En este momento del proceso se establece la necesidad de identificar y dar visibilidad a la pluralidad y heterogeneidad de construcción mentales que los participantes del sistema de acción gestionan sobre los diferentes aspectos del proceso. Más allá de los nodos o «lemas» coincidentes o hegemónicos, es posible identificar otros que cualitativamente los complementan o contradicen (Galtung, 2004; Ibáñez, 1985). Para que resulte realmente un proceso transformador, debe existir un reconocimiento de «todas las voces» $\mathrm{y}$ «todas las direcciones de discurso» a fin de poder abordar un proceso de con- 
sensos y no sólo un proceso de reducciones con base en los trazos mayoritarios o hegemónicos.

- Salto 5: Coproducir con talleres de creatividad social.

Este momento supone un espacio de reflexibilidad de segundo grado, en el que a partir de los elementos identificados en los saltos anteriores y aprovechando las sinergias previamente establecidas es posible ir elaborando «cierres» en cuanto a acuerdos tanto de objetivos como de medios. Supone consolidar trabajos avanzados en fases anteriores y marcar flujos de acción más restrictivos, es decir, es una delimitación del campo, de la dirección y de la forma de acción.

— Salto 6: Planificación de urgencia y a medio plazo.

Supone un salto praxeológico en el que se van avanzando acciones con una doble finalidad, la consecución progresiva de los objetivos planteados y una transformación estructural del sistema en la medida en que se avanza en las ideas-fuerza que sustentan los objetivos operativos y finalistas en sí mismos.

Igualmente supone un empoderamiento progresivo de los diferentes grupos o agentes que participan del proceso en la medida en que se apropian del mismo, lo particularizan en su realidad concreta, pero confirman su capacidad de contribuir al proyecto colectivo.

- Salto 7: La capacidad autoorganizativa de la gente.

Supone una transformación cualitativa del sistema y en sí mismo y de la estructura de relaciones que lo sustenta. Este cambio va orientado por la incorporación de una nueva lógica de proceso, en el que los agentes interactúan por procesos de carácter colaborativo y sinérgico y no sólo por principios de racionalidad instrumental de unos sobre otros.

- Salto 8: Los enfoques transductivos y los desbordes necesarios.

El último salto tiene que ver con la propia naturaleza de los acontecimientos sociales que pueden ser considerados como sistemas de estructura abierta, en la que la culminación de un proceso es el punto de arranque de uno nuevo. En este salto se podría utilizar el símil de una espiral excéntrica de carácter ascendente, en el que el campo de 
acción está delimitado por el área de la espiral sobre la que siempre pivota la dinámica de proceso, pero que está abierta a fenómenos que producen cambios o a una variación de los niveles de acción.

\section{FIGURA 1}

Saltos de los Procesos de Sociopraxis

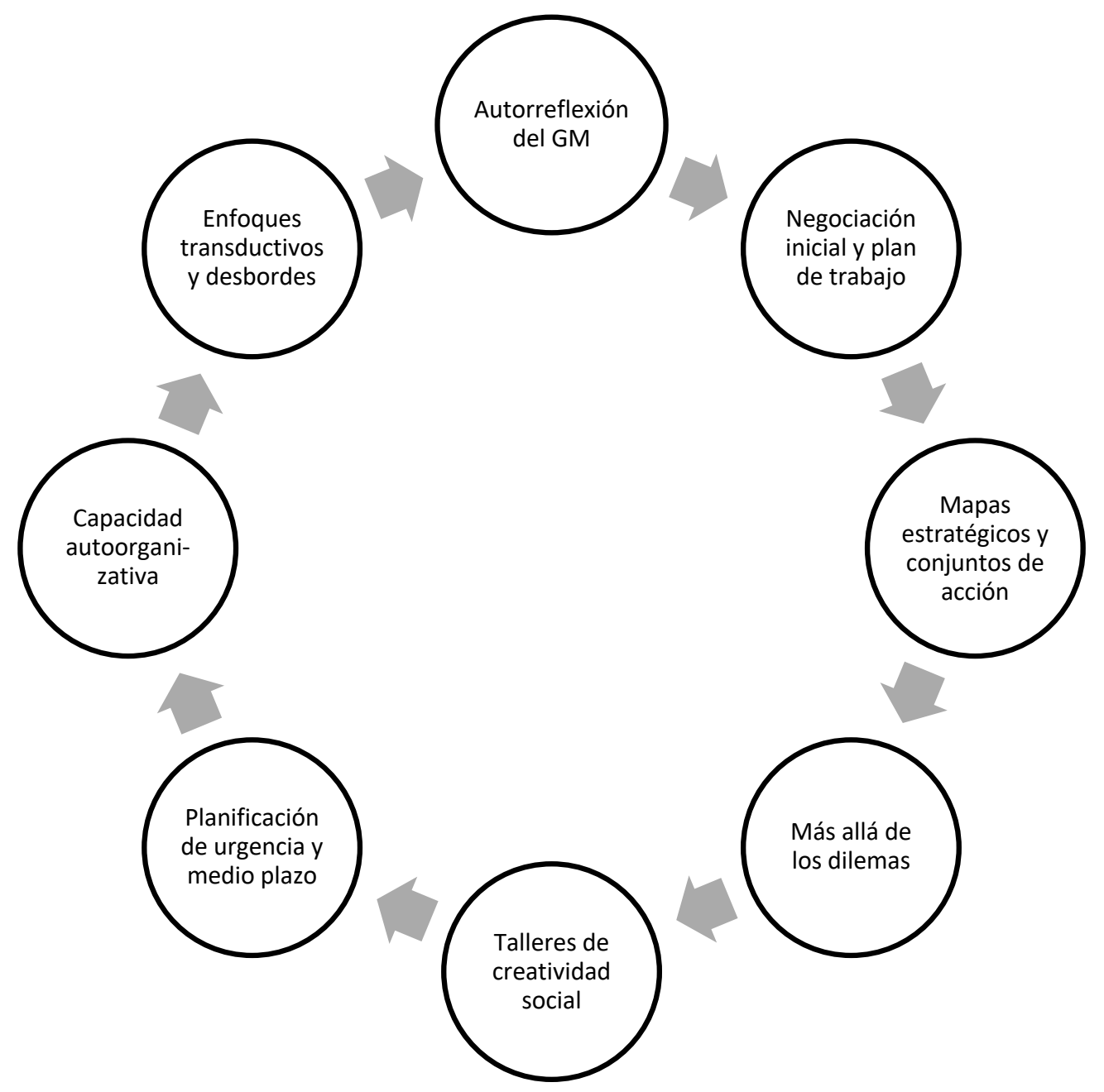

Fuente: Elaboración propia adaptada de (Caballero Ferrándiz et al., 2019). 


\section{EI Modelo Sistemático de Capacidades de Innovación Social}

Si bien es cierto que existe multitud de aportaciones orientadas a intentar definir terminológicamente qué es la innovación social, no ha sido igual el esfuerzo en llevar a cabo modelizaciones más complejas sobre estos fenómenos. Es tan así que resulta realmente complicado encontrar modelos con un cierto nivel de profundidad conceptual y metodológica en sus desarrollos.

Cualquier modelo sobre innovación social debería contar al menos con una serie de elementos mínimos: 1) una definición específica de qué es lo que se considera innovación social; 2) un marco teórico que sustente el modelo; 3 ) una caracterización y relación entre los objetivos a cubrir; 4) una identificación de los actores participantes y la relación entre ellos; 5) una aproximación a los contenidos de las propias prácticas de innovación social; 6) una propuesta de etapas por las que discurre dicho fenómeno; y 7) una delimitación de los resultados esperados en términos de impacto.

Si se utilizara con rigurosidad la necesaria presencia de todos estos elementos para definir un modelo de innovación social, la realidad pondría de manifiesto que no existen realmente modelizaciones sobre el fenómeno de la innovación social.

Es por ello por lo que como referencia de análisis y discusión para este trabajo se va a recurrir al Modelo Sistemático de Capacidades de Innovación Social, que pretende ser un intento aproximativo en esa línea planteada, y recurre para ello al enfoque de la integración sistemática. La propuesta tiene como objetivo proponer un modelo interpretativo de los procesos de innovación social que sea de relevancia práctica para implementar procesos de innovación social intencional. Este modelo se centra en identificar, comprender y explicar las diferentes «capacidades» necesarias que han de encontrarse presentes en una organización compleja de acción social para que emerjan procesos de innovación social.

Al optar por un enfoque de carácter sistemático integrativo, el modelo que se presenta se ve en el reto y en la necesidad de dar cuenta de los diferentes déficits percibidos en las formulaciones teóricas desarrolladas hasta el momento, así como las identificadas en las prácticas mediante la revisión bibliográfica, desarrollando una crítica reflexiva de estos elementos.

Un modelo con aspiraciones de resultar verdaderamente útil debería explicar adecuadamente las diferentes dimensiones de la innovación social y las interrelaciones supuestas entre sí. Entre las dimensiones a clarificar de forma adecuada habría que tener en cuenta: 


\section{(1) Dimensión "Concepto e implicaciones de la innovación social»}

Para este modelo se va a utilizar una definición de innovación social original del autor en términos de «toda producción cultural que surge de un proceso estructurado promovido por un ecosistema de innovación social, que da cobertura de una manera valorable a un acontecimiento social definido bajo el paradigma de los derechos humanos y que además de atender de manera sostenible a la demanda o necesidad que lo origina genera cambios sistémicos significativos».

Como se ve, la definición utilizada es de carácter complejo y relacional, por lo que es preciso hacer un análisis independiente de cada una de las proposiciones utilizadas en su construcción:

- La innovación social como producción cultural: Como se ha visto anteriormente, cada uno de los enfoques teóricos considerados plantean de una manera muy diversa qué sería la concreción de la innovación social. Se identifica un amplio espectro de manifestaciones que pueden ser definidas de manera común como «producciones culturales», entendidas estas como «conjunto de elementos de carácter material, inmaterial, relacional, organizativo o de cualquier otra naturaleza artificial a través de las cuales se puede explicar, interpretar o intervenir los diversos entornos de relaciones a los que están sujetos los seres humanos de manera individual o colectiva»(Herrera Flores, 2005).

- Producto de un proceso estructurado: Una revisión profunda de la literatura muestra una ausencia de manifestaciones en cuanto a establecer la posibilidad de desarrollar procesos de innovación social de carácter intencional, en parte debido a la falta de teorización sobre este aspecto concreto de los procesos de innovación social y de la orientación preferencial hacia los estudios de caso. Sin embargo, hay un cierto consenso por parte de un número de autores que afirman que dicha posibilidad podría darse en aquellas situaciones en las que concurren de una manera precisa una serie de circunstancias y factores. El modelo aquí propuesto se alinea con los estudios que defienden una visión sistemática de los procesos de innovación social, de tal forma que, garantizando la adecuada ejecución de cada una de las fases indicadas, y en presencia del resto de factores facilitadores de proceso, se podría implementar un proceso de innovación social de manera planificada e intencional.

- Promovido por un ecosistema de innovación social: Se entiende por ecosistema de innovación social al sistema resultante de la presencia e interacción entre el conjunto de agentes de innovación social presentes en un entorno entre sí, con 
ellos mismos y con los recursos de diversa naturaleza disponibles en dicho entorno y que tiene como objetivo identificado posibilitar la producción de una innovación social concreta. Los ecosistemas de innovación social son entornos flexibles cuya dinámica es la de tender a maximizar la posibilidad de generar innovaciones sociales y, por lo tanto, su aparición, desarrollo y colapso (o desaparición), que viene determinada en función de la cobertura de la necesidad/ demanda social identificada, es decir, los ecosistemas de innovación social tienen una razón de ser determinados por una necesidad/demanda social concreta, lo que posibilita que en función de la variabilidad de esta necesidad/demanda haya un desarrollo del propio ecosistema.

- Da cobertura de una manera valorable: Una innovación social es una respuesta con capacidad para producir resultados, ya no sólo en términos observables, sino que estos deben tener un carácter de medibles, es decir, que la innovación social debe producir impactos susceptibles de someterse a valoración y cuantificación.

- Acontecimiento social definido bajo el paradigma de los derechos humanos: Se entiende en esta definición los derechos humanos desde una teoría crítica, de tal forma que se éstos se definirían como «medios que en distintos contextos procuran el desarrollo y la apropiación de las capacidades y las potencialidades humanas» (Herrera Flores, 2005). Desde este enfoque, las innovaciones sociales serían producciones culturales que contribuirían al desarrollo del ser humano, ya sea considerado individual o colectivamente, en términos de capacidad y de potencialidad.

- Atiende de manera sostenible y genera cambios sistémicos significativos: Como se ha visto anteriormente, las innovaciones sociales pueden ser consideradas en varios niveles de desarrollo y aplicación. En la mayoría de los casos, estas innovaciones son de corto recorrido y sólo pueden desarrollar impactos elevados en entornos inmediatos, ya que la difusión y la replicación suelen precisar de costes en tiempo y en recursos que superan lo considerado como aceptables por los agentes sociales. Es por ello que va a ser mucho más frecuente la presencia de innovaciones sociales de carácter germinal que de carácter experimentado. Un adecuado proceso de desarrollo de innovaciones sociales de carácter intencional debe tener en cuenta y proveer de los mecanismos y recursos necesarios para posibilitar el tránsito de un tipo de innovaciones sociales al otro, entendiendo que este último puede referirse a sistemas focales, sectoriales u otros de rango más amplio. 


\section{(2) Dimensión «Agentes de innovación social»}

Como se ha podido poner de manifiesto, no se ha identificado de forma unívoca cuál o cuáles son los agentes de innovación social que deberían ser priorizados a la hora de proponer un proceso de innovación social. Lo que sí se puede encontrar es una serie de consensos que se repiten, unas veces como cualidades intrínsecas y otras veces como cualidades deseables. Una sistematización de estos rasgos nos lleva a diferenciar entre:

— agentes primarios de innovación social: serían principalmente aquellos actores con una fuerte orientación hacia lo social como elemento definidor de su identidad y de su razón de ser, con un nivel adecuado de arraigo y especialización en el sistema en el que se encuentra inserto, así como que posee un nivel suficiente de estructuración y estabilidad organizacional como para generar patrones y rutinas a la misma vez que mantiene una dinámica de adaptabilidad y flexibilidad;

- agentes secundarios o auxiliares de innovación social: serían todo el repertorio de actores que participan de un ecosistema de innovación social proveyendo de recursos diferenciales necesarios para la consecución de la innovación social.

\section{(3) Dimensión «Proceso de innovación social»}

El modelo de innovación social aquí propuesto expone una serie de fases que tienen un carácter secuencial no lineal, de tal forma que todas las etapas se han de hacer presentes durante el proceso, pero pueden manifestarse a través de múltiples combinaciones entre las mismas y con una intensidad o duración adaptada a la situación y contexto concretos del proceso que se está desarrollando. Las etapas que considera este modelo son:

- Surgimiento de la necesidad o demanda.

- Delimitación y análisis en profundidad del problema.

- Configuración del ecosistema de innovación.

- Génesis de ideas.

- Proyección e implementación de prototipos.

- Evaluación de resultados primarios, aprendizaje orientado por evidencias y mejora de prototipado.

- Escalamiento, adaptación contextual y evaluación de resultados secundarios.

— Difusión del conocimiento, sostenibilidad y cambio sistémico. 
A continuación se muestra una representación gráfica del modelo presentado.

FIGURA 2

Modelo Sistemático de Capacidades de Innovación Social

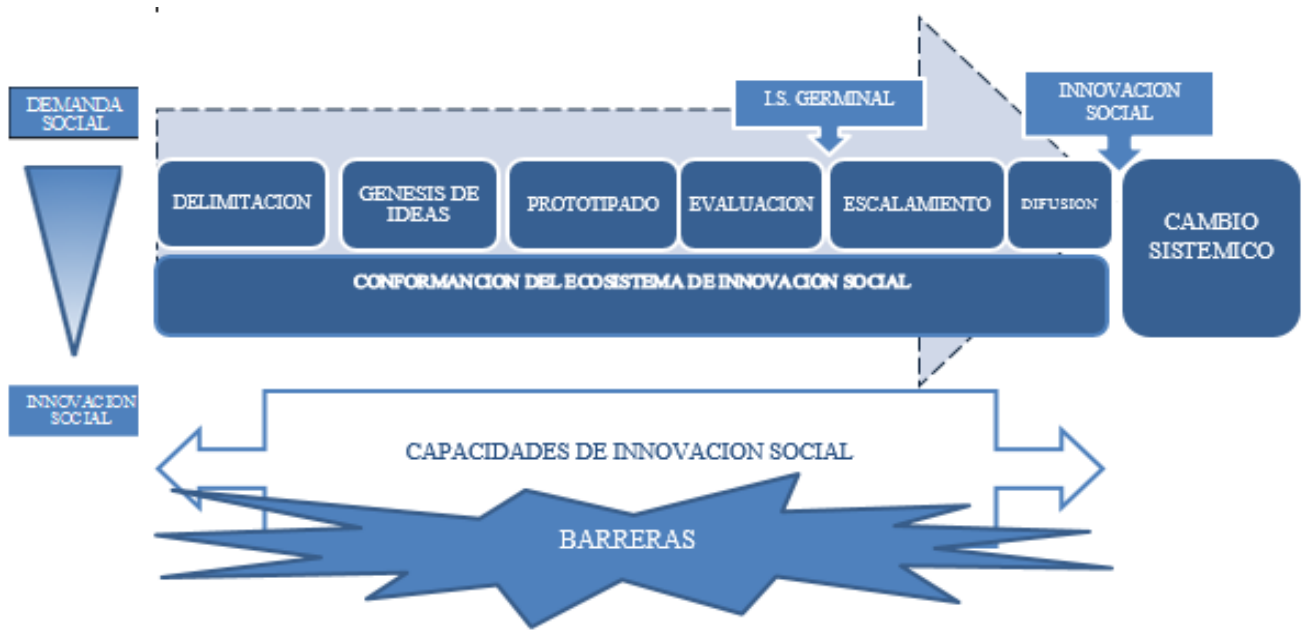

Fuente: Elaboración propia.

Otra de las herramientas conceptuales que se consideran centrales en este modelo. ya que articulan cada uno de los elementos anteriormente descritos (concepto, agentes y procesos de innovación social), es la de «capacidades de innovación social», entendidas éstas como todas aquellas competencias distintivas del proceso de producción de innovaciones sociales cuya aplicación se lleva a cabo en el contexto de los ecosistemas de innovación social mediante la práctica de uno o más agentes y que aseguran el éxito de dichos procesos.

Un análisis más pormenorizado de cada una de estas capacidades no supone materia de este trabajo, por lo que en este momento tan sólo se llevará a cabo una aproximación tentativa a las mismas.

Sobre la base del conjunto de datos obtenidos en el trabajo de Krlev et al. (2014), y complementados mediante la revisión sistemática de la literatura anteriormente expuesta, se escogieron aquellas capacidades, describiendo sus dimensiones e identificando componentes asociados, que se consideraron como las más adecuadas para medir la innovación social. En respuesta a Schibany y Streicher (2008), y siguiendo sus consejos, para evitar una selección arbitraria o ecléctica de elementos, la base para la selección debe apoyarse en su análisis conceptual, por eso los fundamentos precedentes de las innovaciones sociales son tan vitales. 
Es posible establecer, como se ha visto, un total de seis capacidades vinculadas con la innovación social potencial, que se encuadran en cuatro metacapacidades. Junto con estas, es posible identificar otras cuatro capacidades que transmutan la capacidad de innovación social potencial en efectiva.

Cada una de las capacidades vinculadas con la innovación social se articula mediante una serie de componentes, que se presentan brevemente en el siguiente cuadro.

TABLA 1

Capacidades de Innovación Social.

\begin{tabular}{|c|c|c|}
\hline \multicolumn{3}{|c|}{ CAPACIDADES DE INNOVACIÓN SOCIAL POTENCIAL } \\
\hline METACAPACIDAD & CAPACIDAD & DIMENSIÓN \\
\hline \multirow{4}{*}{$\begin{array}{l}\text { CAPACIDAD } \\
\text { COMUNITARIA }\end{array}$} & \multirow{4}{*}{$\begin{array}{l}\text { ORIENTACIÓN HACIA LO } \\
\text { SOCIAL } \\
\text { Este ítem refiere a la incorporación de } \\
\text { la cobertura de necesidades sociales } \\
\text { como un rasgo característico, } \\
\text { distintivo y definitorio de la } \\
\text { organización. }\end{array}$} & Compromiso social \\
\hline & & Refiere al reconocimiento explícito por parte de la \\
\hline & & $\begin{array}{l}\text { organización de promover procesos que repercutan } \\
\text { en una mejora de las condiciones de la sociedad } \\
\text { definidos a partir de parámetros éticos. }\end{array}$ \\
\hline & & $\begin{array}{l}\text { Inversión social } \\
\text { Refiere al porcentaje de recursos de la } \\
\text { organización directamente invertidos en la } \\
\text { producción de beneficios sociales o a la cobertura } \\
\text { de necesidades sociales. }\end{array}$ \\
\hline \multirow{4}{*}{$\begin{array}{l}\text { CAPACIDAD } \\
\text { CÍVICA }\end{array}$} & \multirow{4}{*}{$\begin{array}{l}\text { CAPILARIDAD SOCIAL } \\
\text { Por capilaridad social entendemos la } \\
\text { vinculación de la organización con su } \\
\text { entorno más cercano, el } \\
\text { reconocimiento por parte de la } \\
\text { organización como un actor } \\
\text { significativo dentro del sistema por } \\
\text { parte de dicho entorno, así como la } \\
\text { disponibilidad de capital social por } \\
\text { parte de la organización dentro del } \\
\text { mismo. }\end{array}$} & Vinculación institucional al territorio \\
\hline & & $\begin{array}{l}\text { Refiere al compromiso explícito de la organización } \\
\text { en relación con mejorar las condiciones objetivas } \\
\text { de bienestar del territorio en que se inserta. }\end{array}$ \\
\hline & & $\begin{array}{l}\text { Legitimidad y representación social } \\
\text { Refiere al reconocimiento explícito de la } \\
\text { organización como un actor significativo en el } \\
\text { territorio o en el ámbito en que se sitúa o que } \\
\text { desarrolla su actuación. }\end{array}$ \\
\hline & & $\begin{array}{l}\text { Capital social } \\
\text { Refiere a la disponibilidad de una red amplia de } \\
\text { apoyos recíprocos fácilmente movilizables en el } \\
\text { territorio en que se ubica la organización o en el } \\
\text { ámbito en que desarrolla su actuación. }\end{array}$ \\
\hline CAPACIDAD & \multirow{2}{*}{\multicolumn{2}{|c|}{$\begin{array}{l}\text { GOBERNANZA Y PARTICIPACIÓN } \\
\text { Entendemos la gobernanza como la } \\
\text { habilidad de las relaciones } \\
\text { institucionales para operar como un } \\
\text { actor colectivo en un medio social o } \\
\text { comunidad local. }\end{array}$}} \\
\hline SOCIAL & & \\
\hline
\end{tabular}




\section{CAPACIDADES DE INNOVACIÓN SOCIAL POTENCIAL}

\section{METACAPACIDAD}

CAPACIDAD DE ADAPTACIÓN

\section{CAPACIDAD}

DIMENSIÓN

\section{ORIENTACIÓN HACIA LA} INNOVACIÓN

Por tal idea entendemos la incorporación de la innovación como un rasgo característico, distintivo y definitorio de la organización.
Cultura de innovación

Por este ítem entendemos la presencia de conocimientos, actitudes, prácticas y valores que facilitan la producción o incorporación de elementos de carácter novedoso en la práctica habitual de la organización.

\section{Inversión en innovación}

Hace referencia a la dedicación específica de recursos económicos, humanos o materiales en la producción de innovaciones.

\section{Gestión del cambio}

Este ítem hace referencia a la habilidad de la organización para hacer frente a las tensiones y perturbaciones externas.

\section{GESTION DEL CONOCIMIENTO} Y DEL APRENDIZAJE

En este ítem consideramos el Aprendizaje social

Nos referimos a la construcción de conocimiento derivado de la interacción de un conjunto de desarrollo consciente de procesos y dinámicas que aumenten y mejoren el conocimiento de la organización en áreas críticas de su realidad, así como su capacidad de gestión de estas. actores, que comparten sus visiones y experiencias para desarrollar una visión común de análisis y soluciones, que mejora la capacidad de acción colectiva, la capacidad de resolución de conflictos y alcanza acuerdos cooperativos, y que tiene capacidad transformadora

\section{GESTIÓN CICLO DEL PROCESO}

DE INNOVACIÓN SOCIAL

Este ítem refiere a la aplicación consciente e intencional de los conocimientos, habilidades y destrezas necesarias para desarrollar un proceso de innovación social según cualquiera de los modelos en dicha temática.

\section{Emergencia \\ Hace referencia a la identificación y definición del problema que conlleva la generación de ideas, bienes o procesos de carácter novedoso definidos como posible solución a dichos problemas de una manera planificada.}

\section{Experimentación}

Refiere a la producción, testeo y evaluación orientada a la mejora de las ideas, bienes y procesos generados en la fase anterior.

\section{Apropiación}

Refiere a la incorporación de la idea, bien o servicio mejorado en la fase anterior por parte de otras organizaciones o actores sociales al ser identificado como una mejora competitiva con respecto a otras posibles soluciones en relación con una problemática compartida. 


\section{CAPACIDAD DE INNOVACIÓN SOCIAL EXPERIMENTADA}

\section{CAPACIDAD}

DIMENSIÓN

\section{ESCALABILIDAD}

La escalabilidad puede entenderse como el proceso por el cual una organización que ha validado una forma $o$ modelo para atender un problema social a nivel local diseña una manera de difundirlo en beneficio de otros, procurando maximizar su impacto social para dar acceso a sus productos y servicios a una mayor base de beneficiarios.

INTENSIDAD
Por intensidad entendemos la relación entre inversión y
gasto llevado a cabo en el proceso de producción de
innovación social y número de innovaciones producidas.

\section{Gasto}

Gasto (estimado) para el desarrollo de la innovación (por tipo) como porcentaje del presupuesto total en un periodo considerado.

\section{Eficiencia}

Refiere al menor gasto de recursos por parte de soluciones novedosas para obtener iguales o mejores resultados de forma comparativa que otras soluciones disponibles.

Venta de productos / oferta de servicios

Porcentaje (estimado) de la venta o de ofertas de servicios derivada de una innovación social en un periodo considerado.

Capacidad de producción/incorporación

Porcentaje (estimado) de los servicios o productos producidos / incorporados en un periodo dado y suponen una mejora significativa o una novedad.

\section{Diversidad de producción}

Número absoluto de innovaciones producidas por ámbito de actuación.

\section{INCIDENCIA}

Entendemos por este concepto el proceso deliberado y Capacidad de difusión

Número absoluto de organizaciones que han incorporado la innovación por ámbito de actuación en un periodo dado.

de acciones por parte de la organización dirigidas a influir en otros actores sociales con la finalidad de lograr la incorporación de las innovaciones sociales que producen al identificar que las mismas suponen un beneficio de amplios sectores de la población o a sectores más específicos involucrados en el proceso.

\section{Compatibilidad}

Refiere a la percepción de alineamiento entre la innovación producida y los valores, experiencias, procedimientos, rutinas, conocimientos y habilidades de potenciales organizaciones beneficiarias.

\section{Complejidad}

Refiere a la dificultad de comprensión de la innovación producida por parte de otras potenciales organizaciones beneficiarias. 


\section{CAPACIDAD DE INNOVACIÓN SOCIAL EXPERIMENTADA}

\section{DIMENSIÓN}

Adaptabilidad

Refiere a la percepción de ajuste entre la innovación producida y el contexto de otras potenciales organizaciones beneficiarias.

\section{Multifuncionalidad}

Refiere a la percepción de la posibilidad de adoptar la innovación en diferentes contextos por parte de otras potenciales organizaciones beneficiarias.

\section{Visibilidad}

Refiere a la posibilidad objetiva de observación y acceso de la innovación social por parte de otras potenciales organizaciones beneficiarias.

Aplicabilidad

Refiere a la percepción de utilidad de la innovación social por parte de otras potenciales organizaciones beneficiarias.

Mejora o ventaja relacionada

Refiere a la percepción de una mejora de los resultados directos (eficacia, eficiencia, sostenibilidad impactos y resultados) e indirectos de la innovación social por parte de otras potenciales organizaciones beneficiarias.

\section{IMPACTO}

Por impacto consideramos la mejora significativa y, en algunos casos, perdurable o sustentable en el tiempo, en alguna de las condiciones o características de la población objetivo y que se plantean como esenciales en la definición del problema que dio origen al proceso de innovación.

\section{Alcance de la innovación}

Refiere al número de organizaciones usuarias o beneficiarios de la innovación producida.

Valoración de la innovación

Refiere a la opinión social subjetiva de los sujetos usuarios de la innovación sobre la pertinencia de esta.

Apropiación social de la innovación (utilización) Refiere al porcentaje de sujetos demandantes/necesitados de la innovación social que hacen uso o se ven beneficiados por la misma.

Percepción de los efectos

Opinión subjetiva acerca de la positividad/negatividad de los efectos secundarios de la aplicación de la innovación producida.

Ámbito de eficacia percibido

Refiere a la opinión subjetiva de los usuarios de la innovación social sobre la eficacia de la misma.

Durabilidad del impacto

Refiere a la percepción subjetiva de los usuarios de la innovación social con respecto a la previsión temporal de durabilidad de esta sin necesidad de llevar a cabo nuevas modificaciones.

Fuente: Elaboración propia. 


\section{El proceso en el Modelo Sistemático de Innovación Social como método de sociopraxis y de investigación participativa}

Una vez expuesto de forma sintética el marco analítico propuesto por Caballero Ferrándiz et al. (2019) como estrategia de discriminación de los que procesos que pueden ser considerados como métodos de sociopraxis, se procederá a exponer los principales rasgos a partir de los cuales este trabajo conceptualiza la innovación social y en los que parece posible identificar rasgos propios de un enfoque de sociopraxis. Para ello se discutirá desde dichos parámetros el Modelo Sistemático de Capacidades de Innovación Social.

Como se ha podido ver, pese a la alta variabilidad de contenidos y fines que contempla, integra claramente un primer momento creativo y de generación de conocimiento que posteriormente se ve refutado o verificado en función de su capacidad para responder a las necesidades, las demandas o el contexto que desencadena el proceso de innovación social.

Una primera consideración sería la de tener en cuenta la finalidad sustantiva de la innovación social. En función de los diferentes enfoques, las concreciones de las innovaciones sociales van a tener más un carácter material o inmaterial, van a ser definidos como ideas, servicios o artefactos o van a centrar el foco en las formas de relación o de organización. En resumen, la finalidad sustantiva de la innovación social tiene que ver con la ampliación de los contextos de bienestar comunitario, tal y como se ha podido ver. Esto entronca directamente con la razón de ser de los métodos de sociopraxis.

Manteniendo el foco en los atributos epistemológicos de la innovación social se puede observar que, desde el punto de vista instrumental (es decir, desde los objetivos que persigue), asume plenamente la apreciación hecha por Ortí (1986) de que las metodologías participativas parten de una necesariedad de complemento por deficiencia. Desde este planteamiento, se observa que las metodologías participativas vinculan dos momentos diferentes, y hasta cierto punto en polos alejados, dentro del proceso dentro de generación de conocimiento. En un polo, liderado por la investigación con orientación cualitativa, estaría el contexto de descubrimiento (aquellas actividades exploratorias que contribuyen a la generación de hipótesis), mientras que en el opuesto estaría el contexto de justificación (aquellas actividades capaces de la verificación o falsación de una hipótesis) (Ibáñez, 1985; Cea D’Ancona, 2012).

Estos dos momentos o posiciones se pueden apreciar claramente en la tipología de innovaciones con las que podemos encontrarnos, ya sea en función del mecanismo por 
el que se generan (Innovación Social de primera o de segunda generación) o del momento del proceso en el que aparecen (Innovación Social Potencial e Innovación Social Experimentada).

Frente a las consideraciones hechas sobre la «razón de ser» de la Innovación Social que le permiten alinearse con los métodos de sociopraxis, se van a exponer características relativas a la «forma de ser» de la innovación social como método de sociopraxis.

En primer lugar, se identifica que en los elementos característicos del Salto 1 se encuentran presentes los principales atributos que hacen referencia a la caracterización de los posibles agentes de innovación social, especialmente los de carácter primario, como en las capacidades asociadas a los mismos, tanto específicas como instrumentales, tal y como se ha podido ver. Se hace referencia en este caso a las metacapacidades comunitarias y sociales. Como se ha podido establecer en la presentación del modelo, la propia naturaleza de los agentes de innovación social permite que haya una clara conciencia de la posición en el campo, así como de las construcciones mentales de las que parten, siendo una práctica habitual e interiorizada los procesos de autorreflexión. Como prerrequisito a la innovación social como método de sociopraxis se encuentra la capacidad de conectar y vincularse con las dinámicas sociales.

Al centrar la atención en los Saltos 2 y 3 , se identifica que hay una clara correspondencia con las asunciones de las fases de «Delimitación y análisis en profundidad del problema» $\mathrm{y}$ «Configuración de los ecosistemas de innovación social» que se han expresado en el modelo presentado. Así mismo, se puede comprobar que el modelo identifica estos saltos o momentos diferentes capacidades de carácter cívico y de adaptación especialmente vinculados a la gestión de procesos y conocimientos.

Los Saltos 4, 5 y 6 van a estar fuertemente asociados a las fases de «Génesis de Ideas», «Proyección en Implementación de prototipos», así como de «Evaluación de Resultados Primarios», en las que se van a implementar de forma significativa capacidades que hemos identificado en las esferas de las Capacidades Sociales y Capacidades de Adaptación.

Por su parte, los saltos 7 y 8 presentan una correspondencia con los contenidos de las fases de «Escalamiento»y «Difusión, sostenibilidad y cambio sistémico», fases en las que se ve claramente una presencia de las capacidades de carácter adaptativo, pero también de las capacidades que posibilitan permutar las innovaciones sociales de carácter potencial a su dimensión experimentada. 
TABLA 2

Correspondencia entre Saltos de los Métodos de Sociopraxis y el Modelo Sistemático de Capacidades de Innovación Social

\begin{tabular}{|c|c|c|}
\hline SALTOS & MOMENTOS DEL MSIS & $\begin{array}{c}\text { ATRIBITOS Y CAPACIDADES } \\
\text { DE IS }\end{array}$ \\
\hline Salto 1 & & $\begin{array}{l}\text { Configuración de los actores de } \\
\text { innovación } \\
\text { Orientación hacia lo social }\end{array}$ \\
\hline Salto 2 & \multirow{2}{*}{$\begin{array}{l}\text { Delimitación y análisis en profundidad } \\
\text { Configuración de los ecosistemas de IS }\end{array}$} & \multirow{2}{*}{$\begin{array}{l}\text { Capilaridad social } \\
\text { Gobernanza y participación } \\
\text { Gestión del conocimiento y del } \\
\text { aprendizaje } \\
\text { Gestión del ciclo de innovación }\end{array}$} \\
\hline Salto 3 & & \\
\hline Salto 4 & \multirow{3}{*}{$\begin{array}{l}\text { Génesis de ideas } \\
\text { Proyección e implementación de } \\
\text { prototipos } \\
\text { Evaluación de resultados primarios }\end{array}$} & \multirow{3}{*}{$\begin{array}{l}\text { Gobernanza y participación } \\
\text { Gestión del conocimiento y del } \\
\text { aprendizaje } \\
\text { Gestión del ciclo de innovación }\end{array}$} \\
\hline Salto 5 & & \\
\hline Salto 6 & & \\
\hline Salto 7 & \multirow{2}{*}{$\begin{array}{l}\text { Escalamiento } \\
\text { Difusión, sostenibilidad y cambio } \\
\text { sistémico }\end{array}$} & Escalabilidad \\
\hline Salto 8 & & $\begin{array}{l}\text { Intensidad } \\
\text { Incidencia } \\
\text { Impacto }\end{array}$ \\
\hline
\end{tabular}

Fuente: Elaboración propia.

\section{CONCLUSIONES}

Como se ha ido viendo a lo largo de todo trabajo, existe un interés creciente en entender, explicar y hasta cierto punto poder gestionar de manera planificada los procesos de innovación social tanto desde el ámbito de las prácticas públicas como de la curiosidad académico-científica (Van der Have y Rubalcaba, 2016; Avelino et al., 2017). No existen dudas del carácter y de la utilidad praxeológica de estos fenómenos, que se entienden como importantes recursos a partir de los cuales afrontar los desafíos sociales, sea cual sea su naturaleza, gravedad o ámbito.

Frente a esta certeza praxeológica existe el reconocimiento de que la innovación social precisa un desarrollo más profundo como ámbito de investigación, ya que en la actualidad aún no es posible identificar un cuerpo de estudio más allá de bloque emer- 
gente de teorías y prácticas con orígenes muy diferentes en las diferentes ciencias sociales (Westley, 2013; Van der Have y Rubalcaba, 2016). Este escenario, caracterizado por unas altas expectativas generadas en torno a este tipo de procesos, el reconocimiento de su capacidad potencial y la diversidad de manifestaciones bajo las que se presentan, justifican la necesidad urgente de llevar a cabo un trabajo en el ámbito de la generación de marcos teóricos que permita promover consensos y unificar posiciones a fin de dar mayor robustez a las actuaciones llevadas a cabo en el ámbito de la investigación, de la política y de la práctica social (Westley, 2013; Haxeltine et al., 2013; Cajaiba-Santana, 2014; Van der Have y Rubalcaba, 2016).

Es en esta necesidad donde surge la oportunidad de proponer nuevas aproximaciones al estudio de este fenómeno. Más allá de plantear la innovación social como un conjunto de metaasunciones, elementos explicativos $\mathrm{u}$ objetos de estudio, se abre la oportunidad de revisar los planteamientos sobre esta materia aplicando el filtro de poder ser conceptualizada más que una forma sustantiva, es decir, de contenidos, de una forma praxeológica.

Como se ha podido observar, se puede afirmar que la innovación social ha ido evolucionando de tal forma que ha ido adquiriendo rasgos propios de un método de sociopraxis, si bien no se ha consolidado de una forma sistemática. La disparidad y la pluralidad de puntos de partida, tanto epistemológicos como metodológicos, en el ámbito de la innovación social, han sido una de las principales dificultades para que no se haya sistematizado y consolidado hasta el momento una propuesta de la innovación social como método especifico de sociopraxis social, con especial incidencia en la producción de productos culturales de alto impacto social, aunque sí dispone de una capacidad potencial para ello.

Siguiendo el marco analítico propuesto por (Caballero Ferrándiz et al., 2019), vemos cómo la innovación social expresada a partir del Modelo Sistemático de Capacidades de Innovación Social tiene robustez y coherencia suficiente para poder ser asumida como un método de sociopraxis, aunque presentando una serie de particularidades y especificidades que la dotan de singularidad con respecto a otros métodos de este mismo enfoque, y precisando aún de un profundo trabajo de fundamentación y formulación teórico-práctica.

\section{BIBLIOGRAFÍA}

Adams, D., \& Hess, M. (2010). «Social Innovation and Why it Has Policy Significance». The Economic and Labour Relations Review, 21(2), 139-155. https://doi. org/10.1177/103530461002100209 
Anderson, T., Curtis, A., \& Wittig, C. (2014). Definition and theory in social innovation. Danube University Krems.

Avelino, F., Wittmayer, J., Pel, B., Weaver, P., Dumitru, A., Haxeltine, A., \& O'Riordan, T. (2017). «Transformative social innovation and (dis) empowerment». Technological Forecasting and Social Change.

Bassi, A. (2011). «Social innovation: Some definitions». Boletín del Centro de Investigación de Economía y Sociedad, CIES, 8.

Bignetti, L. P. (2011). «As inovações sociais: uma incursão por ideias, tendências e focos de pesquisa. Social innovation: ideas, tendencies and research possibilities». Ciências Sociais Unisinos, 47(1), 3-14. https://doi.org/10.4013/csu.2011.47.1.01

Caballero Ferrándiz, J., Martín Gutiérrez, P., \& Villasante, T. R. (2019). «Debatiendo las metodologías participativas: Un proceso en ocho saltos». EMPIRIA. Revista de $\mathrm{Me}-$ todología de Ciencias Sociales (44), 21-45. https://doi.org/empiria.43.2019.25350

Cajaiba-Santana, G. (2014). «Social innovation: Moving the field forward. A conceptual framework». Technological Forecasting and Social Change, 82, 42-51. https://doi. org/10.1016/j.techfore.2013.05.008

Callejo, J., y Viedma, A. (2006). Proyectos y estrategias de investigación social: la perspectiva de la intervención. Madrid: McGraw Hill.

Callejo, J., y Viedma, A. (2015). «La investigación-acción participativa». En M. Ferrando, García, F. Alvira, L. E. Alonso, \& M. Escobar (eds.), El análisis de la realidad social: métodos y técnicas de investigación (pp. 295-328). Madrid: Alianza Editorial.

Cea D'Ancona, M. A. (2012). Fundamentos y aplicaciones de la metodología cuantitativa. Madrid: Editorial Síntesis.

Cembranos, F., \& Medina, J. A. (2003). Grupos inteligentes: teoría y práctica del trabajo en equipo. Madrid: Editorial Popular.

Chaves, L. (2007). «El paradigma cualitativo en la investigación educativa: una aproximación teórica». En: Chaves, L., Díaz, M., García, J., Rojas, G., Solís, N. (eds.) (2007). Investigación-acción colaborativa: un encuentro con el quehacer cotidiano del centro educativo para su trasformación. San José, Costa Rica: Instituto de Investigación en Educación y Universidad de Costa Rica.

Cimas, R. (2015). Metodologías participativas. Sociopraxis para la creatividad social. Madrid: DEXTRA.

Colas Bravo, M. (1998). «Métodos y técnicas cualitativas de investigación en psicopedagogía». En Colás-Bravo, M., Buendía, L., Hernández, F. (eds.) (1998), Métodos de investigación en psicopedagogía. Madrid, España: Editorial McGraw Hill Latinoamericana. 
Cunha, J., \& Benneworth, P. (2013). «Universities' contributions to social innovation: towards a theoretical framework». En EURA Conference. https://doi.org/10.1051/ bioconf $/ 201101000$

Denyer, D., y Neely, A. (2004). «Introduction to special issue: Innovation and productivity performance in the UK». International Journal of Management Reviews, 5, 131-135.

Edwards-Schachter, M. E., Matti, C. E., \& Alcántara, E. (2012). «Fostering Quality of Life through Social Innovation: A Living Lab Methodology Study Case». Review of Policy Research, 29(6), 672-692. https://doi.org/10.1111/j.1541-1338.2012.00588.x

Galtung, J. (2004). Transcender y transformar. Una introducción al trabajo de conflictos. México, DF: Editorial Quimera.

Haxeltine, A., Wittmayer, J., Avelino, F., Kemp, R., Weaver, P., Backhaus, J., \& O’ Riordan, T. (2013). «Transformative social innovations: A sustainability transition perspective on social innovation». Social Frontiers. The next edge of social innovation research. https://doi.org/10.1006/jabr.1997.7032

Hernández, J., Tirado, P., \& Ariza, A. (2016). «El Concepto de Innovación Social:Ámbitos,Definiciones y Alcances teóricos». CIRIEC-España, Revista de Economía Pública, Social y Cooperativa.

Herrera Flores, J. (2005). Los derechos humanos como productos culturales. Crítica del humanismo abstracto. Madrid: Libros de la Catarata.

Hochgerner, J. (2011). The analysis of social innovations as social practice. Bridges, 30 . Howaldt, J., \& Schwarz, M. (2010). «Social Innovation: Concepts, research fields and international trends». Dortmund: Report of ESF, EU, and Aachen University. https:// doi.org/10.1007/978-3-642-36540-9

Hubert, A. (2010). «Empowering people, driving change. Social innovation in the European Union». Bepa. https://doi.org/10.2796/13155

Ibáñez, J. (1985). Del algoritmo al sujeto. Perspectivas de la investigación social. Madrid: Siglo XXI Editores.

Jessop, B., Moulaert, F., Hulgard, L., \& Hamdouch, A. (2013). «Social innovation research: a new stage in innovation analysis?». En F. Moulaert (Ed.) et. al., The International Handbook on Social Innovation. Cheltenham: Edward Elgar Publishing.

Kirwan, J., Ilbery, B., Maye, D., \& Carey, J. (2013). «Grassroots social innovations and food localisation: An investigation of the Local Food programme in England». Global Environmental Change, 23(5), 830-837. https://doi.org/10.1016/j.gloenvcha.2012.12.004 
Krlev, G., Bund, E., \& Mildenberger, G. (2014). «Measuring What Matters-Indicators of Social Innovativeness on the National Level». Information Systems Management. https://doi.org/10.1080/10580530.2014.923265

Marcy, R. T., \& Mumford, M. D. (2007). «Social innovation: Enhancing creative performance through causal analysis». Creativity Research Journal. https://doi. org/10.1080/10400410701397180

Marques, C. S., Gerry, C., Diniz, F., \& Ferreira, A. L. (2012). «Social innovation: determinants of the demand for high-quality institutional care by the elderly». Journal of Knowledge Management, Economics and Information Technology, 2(2), 186-202.

Moulaert, F. \& Van Dyck, B. (2013). «Framing social innovation research: a sociology of knowledge perspective». En International Handbook on Social Innovation.

Moulaert, F., \& Nussbaumer, J. (2005). «Defining the Social Economy and its Governance at the Neighbourhood Level: A Methodological Reflection». Urban Studies, 42(11), 2071-2088. https://doi.org/10.1080/420980500279752

Murray, R., Caulier-Grice, J., \& Mulgan, G. (2010). «The Open Book of Social Innovation». En The Young Foundation. https://doi.org/10.1371/journal.pcbi.0030166

Neumeier, S. (2012). «Why do Social Innovations in Rural Development Matter and Should They be Considered More Seriously in Rural Development Research? - Proposal for a Stronger Focus on Social Innovations in Rural Development Research». Sociologia Ruralis, 52(1), 48-69. https://doi.org/10.1111/j.1467-9523.2011.00553.x Nicholls, A., \& Murdock, A. (2012). «Social Innovation». En A. Nicholls \& A. Murdock (eds.), Social Innovation: Blurring Boundaries to Reconfigure Markets. https://doi. org/10.1057/9780230367098

Nicholls, A., Simon, J., \& Gabriel, M. (2016). «New Frontiers in Social Innovation Research». En New Frontiers in Social Innovation Research. https://doi. org/10.1007/978-1-137-50680-1

Ortí, A. (1986). «La apertura y el enfoque cualitativo o estructural: la entrevista abierta semidirectiva y la discusión de grupo». En M. García Ferrando, J. Ibáñez, \& F. Alvira (eds.), El análisis de la realidad social (pp. 156-186). Madrid: Alianza Universidad Textos.

Pittaway, L., Robertson, M., Munir, K., Denyer, D., \& Neely, A. (2004). Networking and innovation: A systematic review of the evidence. Journal of Management Reviews, 5-6, 137-168.

Portes, A. (2013). Sociología económica. Madrid: Centro de Investigaciones Sociológicas. 
Pot, F., \& Vaas, F. (2008). «Social innovation, the new challenge for Europe». International Journal of Productivity and Performance Management, 57(6), 468-473. https://doi.org/10.1108/17410400810893400

Pue, K., Vandergeest, C., \& Breznitz, D. (2016). Toward a theory of social innovation. Toronto: University of Toronto.

Schibany, A., \& Streicher, G. (2008). «The European innovation scoreboard: Drowning by numbers?». Science and Public Policy, 35(10), 717-732.

Subirats, J. \& García Bernardos, A. (2015). Innovación social y políticas urbanas en España. Barcelona: Icaria.

Tepsie (2014). «Social innovation theory A Guide for Researchers». Tepsie. https://doi. org/10.1021/acs.analchem. $7 \mathrm{~b} 01798$

- (2014). «Social Innovation Theory and Research: A Summary of the Findings from TEPSIE». En A deliverable of the project: «The theoretical, empirical and policy foundations for building social innovation in Europe» (TEPSIE), European Commission - 7th Framework Programme, Brussels: European Commission, DG Research. https://doi.org/10.1021/acs.analchem.7b01798

Tranfield, D., Denyer, D., \& Smart, P. (2003). «Towards a methodology for developing evidence-informed management knowledge by means of systematic review». British Journal of Management, 14, 207-222.

Van der Have, R. P., \& Rubalcaba, L. (2016). «Social innovation research: An emerging area of innovation studies?». Research Policy, 45(9), 1923-1935. https://doi.or$\mathrm{g} / 10.1016 / \mathrm{j}$.respol.2016.06.010

Vercauteren, D., Mouss Crabbé, O., \& Muller, T. (2010). Micropolíticas de los grupos para una ecología de las prácticas colectivas. Madrid: Editorial Traficantes de Sueños.

Wang, H., \& Wang, C. (2003). «Taxonomy of security considerations and software quality». Communications of the ACM, 46(6), 75-78. https://doi.org/10.1145/777313. 777315

Westley, F. (2013). «Social Innovation and Resilience: How One Enhances the Other». Stanford Social Innovation Review, Summer, 6-8.

Villasante, T. (1995). «De los movimientos sociales a las metodologías participativas». En: Delgado, J. y Gutiérrez, J. (eds.), Métodos y Técnicas Cualitativas de Investigación en Ciencias Sociales. Madrid, España: Editorial Síntesis. 